\title{
Avaliação ultra-sonográfica da mobilidade do diafragma em diferentes posturas em sujeitos saudáveis*
}

\author{
Ultrasound evaluation of diaphragmatic mobility \\ in different postures in healthy subjects

\begin{abstract}
Wellington Pereira dos Santos Yamaguti ${ }^{1}$, Elaine Paulin ${ }^{2}$, Simone Shibao ${ }^{3}$, Sérgio Kodaira ${ }^{3}$, Maria Cristina Chammas ${ }^{4}$, Celso Ricardo Fernandes Carvalho ${ }^{5}$
\end{abstract}

\begin{abstract}
Resumo
Objetivo: Avaliar, por meio de ultra-sonografia, o efeito da mudança de posicionamento corporal na mobilidade diafragmática de sujeitos saudáveis durante a respiração espontânea. Métodos: Foram avaliadas sete mulheres voluntárias, saudáveis, sem histórico clínico de doenças cárdio-respiratórias, não tabagistas e eutróficas. Os sujeitos foram submetidos à prova de função pulmonar e à avaliação ultra-sonográfica da mobilidade do diafragma direito através do deslocamento crânio-caudal do ramo esquerdo da veia porta, utilizando-se um aparelho de ultra-sonografia no modo B. A mobilidade do diafragma direito foi avaliada em decúbito lateral direito e em decúbito lateral esquerdo, e a ordem de avaliação das posturas foi definida aleatoriamente por meio de um sorteio prévio. Resultados: A média da excursão do diafragma direito em decúbito lateral direito $(51,30 \pm 9,69 \mathrm{~mm})$ foi significativamente maior $(\mathrm{p}=0,03)$ que em decúbito lateral esquerdo $(45,93 \pm$ $10,37 \mathrm{~mm}$ ). Conclusão: Os resultados sugerem que, durante a ventilação espontânea, a mobilidade da porção dependente do diafragma é maior do que a da não dependente e que a técnica empregada foi sensível para detectar variações da mobilidade do diafragma com as mudanças de posicionamento.
\end{abstract}

Descritores: Diafragma; Ultra-sonografia; Testes de função respiratória; Postura.

\begin{abstract}
Objective: To assess, using ultrasound, the effects that changes in body position have on diaphragmatic mobility in healthy subjects during spontaneous breathing. Methods: The study involved seven healthy female volunteers, all of whom were nonsmokers, well nourished, and free of any cardiopulmonary disease. They were submitted to pulmonary function testing and ultrasound evaluation of the mobility of the right diaphragm by the craniocaudal displacement of the left branch of the portal vein using an ultrasound device in mode B. The mobility of the right diaphragm was evaluated in right decubitus and in left decubitus. The order of evaluation was previously determined in a random drawing. Results: The average mobility of the right diaphragm in right decubitus ( $51.30 \pm 9.69 \mathrm{~mm})$ was significantly higher ( $p=0.03)$ than that observed in left decubitus (45.93 $\pm 10.37 \mathrm{~mm}$ ). Conclusion: The results suggest that, during spontaneous ventilation, the dependent portion of the diaphragm presents greater mobility than does the nondependent portion, and that the technique used was sufficiently sensitive to detect variations in diaphragmatic mobility related to changes in posture.
\end{abstract}

Keywords: Diaphragm; Ultrasonography; Respiratory function tests; Posture.

\footnotetext{
* Trabalho realizado na Faculdade de Medicina da Universidade de São Paulo - FMUSP - São Paulo (SP) Brasil.

1. Mestre em Ciências da Reabilitação. Faculdade de Medicina da Universidade de São Paulo - FMUSP - São Paulo (SP) Brasil.

2. Doutora em Ciências. Faculdade de Medicina da Universidade de São Paulo - FMUSP - São Paulo (SP) Brasil.

3. Médica Radiologista do Instituto de Radiologia do Hospital das Clínicas. Faculdade de Medicina da Universidade de São Paulo - FMUSP - São Paulo (SP) Brasil.

4. Doutora em Radiologia. Faculdade de Medicina da Universidade de São Paulo - FMUSP - São Paulo (SP) Brasil.

5. Livre Docente em Fisioterapia. Faculdade de Medicina da Universidade de São Paulo - FMUSP - São Paulo (SP) Brasil.

Endereço para correspondência: Celso Ricardo Fernandes de Carvalho. Av. Dr. Arnaldo, 455, sala 1216, CEP: 05408-040, São Paulo, SP, Brasil.

Tel 5511 3066-7317.Fax 5511 3091-7462.E-mail: cscarval@usp.brou wellpsy@yahoo.com.br

Recebido para publicação em 12/8/06. Aprovado, após revisão, em 14/11/06.
} 


\section{Introdução}

0 posicionamento corporal é considerado uma intervenção terapêutica não invasiva, com efeitos significativos e benéficos sobre a função pulmonar e o transporte de oxigênio, interferindo na distribuição da ventilação, da perfusão, da pressão de abertura dos alvéolos e na mecânica diafragmática. ${ }^{(1)}$

Diferenças no padrão de movimento do diafragma têm sido observadas em indivíduos saudáveis durante a ventilação mecânica com pressão positiva e/ou durante a respiração espontânea em diferentes posturas. ${ }^{(2,3)}$ Alguns autores constataram que, durante a ventilação mecânica, a mobilidade do diafragma é significativamente maior nas zonas não dependentes e que, durante a respiração espontânea, o padrão de mobilidade do diafragma é maior nas zonas dependentes, onde a força de oposição ao deslocamento diafragmático (a pressão hidrostática do abdômen) tende a ser maior. ${ }^{(2)}$

A avaliação da mobilidade do diafragma tem sido tradicionalmente realizada pela fluoroscopia. ${ }^{(4)}$ Apesar de esse método ser considerado o padrãoouro, ele apresenta algumas limitações, tais como visualização do diafragma através de uma única incidência, necessidade de cálculos corretivos e exposição do paciente à radiação ionizante. ${ }^{(5)}$ Nos últimos anos, a ultra-sonografia também passou a ser utilizada na avaliação da mobilidade do diafragma por apresentar algumas vantagens em relação à fluoroscopia: portabilidade; ser desprovida de radiação ionizante; e por quantificar diretamente o movimento diafragmático. ${ }^{(6-10)}$

Apesar das vantagens da ultra-sonografia, a visualização direta do diafragma apresenta dificuldades metodológicas que dependem do posicionamento do transdutor. ${ }^{(7)}$ Recentemente, foi validado um método ultra-sonográfico que avalia a mobilidade do diafragma direito por meio de incidência do feixe sonoro perpendicular ao eixo crânio-caudal utilizando a janela abdominal subcostal para a visualização do deslocamento do ramo esquerdo da veia porta do fígado como medida indireta do deslocamento do hemidiafragma direito. ${ }^{(11)}$

0 objetivo do presente estudo foi avaliar o efeito da mudança de posicionamento corporal na mobilidade diafragmática em indivíduos saudáveis durante a respiração espontânea utilizando esse novo método de avaliação ultra-sonográfica.

\section{Métodos}

\section{Sujeitos e casuística}

Este estudo foi observacional e transversal e os dados foram coletados no Instituto de Radiologia do Hospital das Clínicas da Faculdade de Medicina da Universidade de São Paulo. Foram avaliadas sete mulheres voluntárias, saudáveis, sem histórico clínico de doenças cárdio-respiratórias, não tabagistas e eutróficas $\left(18,5 \mathrm{~kg} / \mathrm{m}^{2}<\right.$ índice de massa corpórea $\left.<25 \mathrm{~kg} / \mathrm{m}^{2}\right)$. Os sujeitos da pesquisa foram submetidos à prova de função pulmonar e à avaliação ultra-sonográfica no mesmo dia. 0 estudo foi previamente aprovado pelo Comitê de Ética do Hospital sob o número 914/04 e todas as voluntárias que participaram assinaram o termo de consentimento livre e esclarecido.

\section{Parâmetros analisados}

Avaliação da função pulmonar: a espirometria (Microquark; Cosmed, Roma, Itália) foi realizada seguindo as normas preconizadas pela American Thoracic Society e pelo 1 Consenso Brasileiro de Espirometria. ${ }^{(12,13)}$ Os sujeitos foram orientados a repousar por cinco a dez minutos antes do teste e os procedimentos a serem realizados foram descritos cuidadosamente, dando ênfase à necessidade de se evitar vazamentos em torno da peça bucal e realizar uma inspiração máxima seguida de expiração máxima e sustentada, até que o avaliador ordenasse a interrupção. 0 ambiente de realização dos exames era calmo e todos os testes foram realizados no período matutino para evitar as influências circadianas. Os sujeitos foram orientados a permanecer sentados e utilizar um clipe nasal durante a realização dos testes. As curvas volume-tempo e fluxo-volume foram realizadas de acordo com os critérios de aceitabilidade preconizados pela American Thoracic Society, ${ }^{(12)}$ sendo escolhida a melhor de três curvas reprodutíveis (variabilidade $<5 \%$ ). A partir dessa curva foram computados os valores da capacidade vital forçada (CVF) e volume expiratório forçado no primeiro segundo $\left(\mathrm{VEF}_{1}\right)$, os quais foram analisados segundo os valores previstos por alguns autores. ${ }^{(14)}$

\section{Análise ultra-sonográfica}

A mobilidade do diafragma direito foi avaliada pelo deslocamento crânio-caudal do ramo esquerdo 
da veia porta utilizando-se um aparelho de ultrasonografia (Logic 500, Pro Series ${ }^{\circledR}$; General Electric Medical Systems, Milwaukee, WI, USA) no modo B. ${ }^{(11)}$ 0 ultra-sonografista utilizou um transdutor convexo de $3,5 \mathrm{MHz}$ posicionado na região subcostal direita, com incidência perpendicular ao eixo crânio-caudal, na direção da veia cava inferior. A seguir, era identificado um ramo portal intra-parenquimatoso no campo de visão e a sua posição era demarcada com o cursor durante a expiração e inspiração forçadas, sendo o deslocamento crânio-caudal desses pontos considerado como o valor da mobilidade diafragmática direita (Figura 1). A mobilidade do diafragma direito foi avaliada em decúbito lateral direito (DLD) e em decúbito lateral esquerdo (DLE), e a ordem de avaliação das posturas foi definida através de randomização, por meio de um sorteio prévio. Os testes foram sempre realizados pelo mesmo radiologista, que desconhecia os valores da função pulmonar das voluntárias e o objetivo do estudo. Foram realizadas três mensurações para cada posição e o maior valor foi selecionado para análise estatística.

\section{Análise estatística}

A distribuição dos dados foi avaliada utilizandose o teste de Kolmogorov-Smirnov e o tamanho amostral de sete sujeitos foi calculado de acordo com os seguintes pressupostos: (i) análise por teste t pareado para medidas repetidas; (ii) variação de

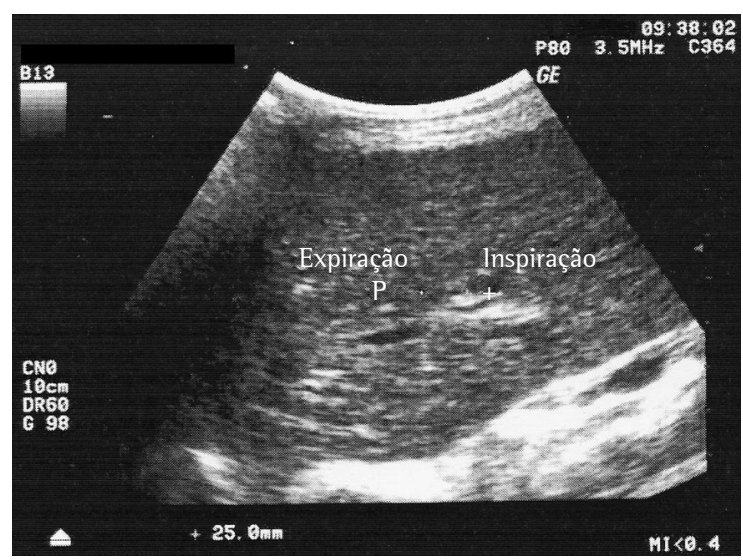

Figura 1 - Avaliação ultra-sonográfica do deslocamento crânio-caudal de um ramo portal intra-hepático esquerdo. A posição do vaso foi marcada pelo caliper em expiração e inspiração forçadas. 0 deslocamento crânio-caudal desses pontos foi registrado em milímetros e considerado como valor da mobilidade do diafragma direito.
45\% na mobilidade do diafragma com os diferentes posicionamentos $^{(2)}$; (iii) desvio padrão de $25 \%$ na mobilidade diafragmática entre sujeitos ${ }^{(2)}$; e (iv) poder de determinação de $80 \%$, que estabeleceu uma amostra de quatro sujeitos. 0 nível de significância foi ajustado para uma variação menor que 5\% ( $p<0,05)$ (Statistical Package Sigma Stat 3.1).

\section{Resultados}

As características antropométricas e a função pulmonar dos sujeitos estão descritas na Tabela 1, em que se verifica função pulmonar dentro de valores considerados normais $\left(\mathrm{VEF}_{1}=110,00 \pm\right.$ $11,02 \%$ do previsto e CVF $=107,84 \pm 16,03 \%$ do previsto). A Tabela 2 ilustra dados individuais referentes ao índice de massa corpórea, parâmetros da função pulmonar e mobilidade do diafragma direito mensurada em DLE e DLD.

Todos os sujeitos estudados apresentaram maior mobilidade do diafragma direito quando avaliados em DLD (decúbito dependente) (Figura 2). Verificou-se ainda que a maior variação da mobilidade diafragmática entre os decúbitos foi de $26,76 \%$ (sujeito 3) e a menor variação foi de $0,46 \%$ (sujeito 1).

A Figura 3 apresenta a média dos valores da mobilidade do diafragma direito em DLD (51,30 \pm 9,69 mm) e DLE (45,93 $\pm 10,37 \mathrm{~mm})$. A média da excursão do diafragma direito foi significativamente maior quando os sujeitos foram posicionados em $\operatorname{DLD}(p=0,03)$. Ao analisar a variação da média da mobilidade diafragmática nos diferentes decúbitos, verifica-se que essa variação foi de 10,47\%.

\section{Discussão}

No presente estudo, a mobilidade do diafragma direito foi mensurada em DLD (decúbito dependente)

Tabela 1 - Características antropométricas e função pulmonar dos sujeitos participantes.

\begin{tabular}{lc}
\hline \multicolumn{1}{c}{ Variável } & Sujeitos $(\mathrm{n}=7)$ \\
\hline ldade (anos) & $24,25 \pm 2,65$ \\
$\mathrm{IMC}\left(\mathrm{kg} / \mathrm{m}^{2}\right)$ & $20,95 \pm 2,81$ \\
$\mathrm{VEF}_{1}(\%$ do previsto) & $110,00 \pm 11,02$ \\
$\mathrm{CVF}(\%$ do previsto) & $107,84 \pm 16,03$ \\
$\mathrm{VEF}_{1} / \mathrm{CVF}(\%)$ & $106,14 \pm 5,97$ \\
\hline
\end{tabular}

Valores expressos pela média \pm desvio padrão; IMC: índice de massa corpórea; $\mathrm{VEF}_{1}$ : volume expiratório forçado no primeiro segundo; e CVF: capacidade vital forçada. 
Tabela 2 - Índice de massa corpórea, função pulmonar e mobilidade do diafragma direito dos sujeitos estudados.

\begin{tabular}{lccccc}
\hline & $\mathrm{IMC}\left(\mathrm{kg} / \mathrm{m}^{2}\right)$ & $\mathrm{VEF}_{1}$ (\% do previsto) & CVF (\% do previsto) & MDD em DLE $(\mathrm{mm})$ & MDD em DLD (mm) \\
\hline Sujeito 1 & 24,9 & 129,9 & 123,1 & 64,6 & 64,9 \\
Sujeito 2 & 23,03 & 105,1 & 113,6 & 46,4 & 60,2 \\
Sujeito 3 & 16,61 & 115,4 & 116 & 42,7 & 58,3 \\
Sujeito 4 & 22,31 & 102,7 & 100,8 & 51,8 & 53,2 \\
Sujeito 5 & 19,29 & 97,6 & 99,8 & 37,2 & 44,7 \\
Sujeito 6 & 19,03 & 94,8 & 95,6 & 39,7 & 41,3 \\
Sujeito 7 & 21,48 & 95,2 & 87,4 & 32 & 37,3 \\
\hline
\end{tabular}

IMC: índice de massa corpórea; $\mathrm{VEF}_{1}$ : volume expiratório forçado no primeiro segundo; CVF: capacidade vital forçada; MDD mobilidade do diafragma direito; DLE: decúbito lateral esquerdo; e DLD: decúbito lateral direito.

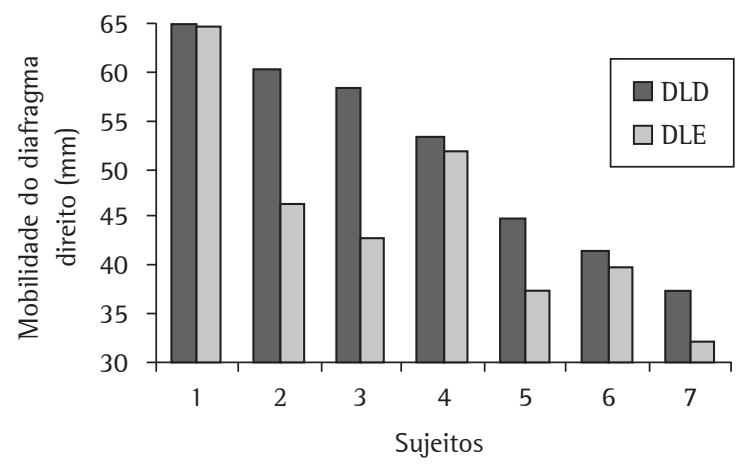

Figura 2 - Avaliação da mobilidade do diafragma direito de sete sujeitos saudáveis posicionados em decúbito lateral direito (DLD, decúbito dependente) e decúbito lateral esquerdo (DLE, decúbito não dependente). Notase que todos os sujeitos apresentam maior mobilidade do diafragma direito quando posicionados sobre o decúbito dependente.

e em DLE (decúbito não dependente) utilizando uma nova técnica de avaliação ultra-sonográfica. Os resultados mostraram que o diafragma direito apresenta maior mobilidade quando os indivíduos estão posicionados sobre o decúbito dependente.

Alguns autores avaliaram a mobilidade diafragmática utilizando a fluoroscopia em três sujeitos saudáveis posicionados tanto em supino como em decúbitos laterais e verificaram que as porções dependentes do diafragma apresentam maior deslocamento na direção crânio-caudal do que as porções não dependentes, ${ }^{(2)}$ corroborando os resultados deste estudo. Os autores discutem que o maior deslocamento da porção dependente do diafragma pode acontecer devido à maior força de oposição ao deslocamento do músculo (a pressão hidrostática do abdômen) presente nessa região.

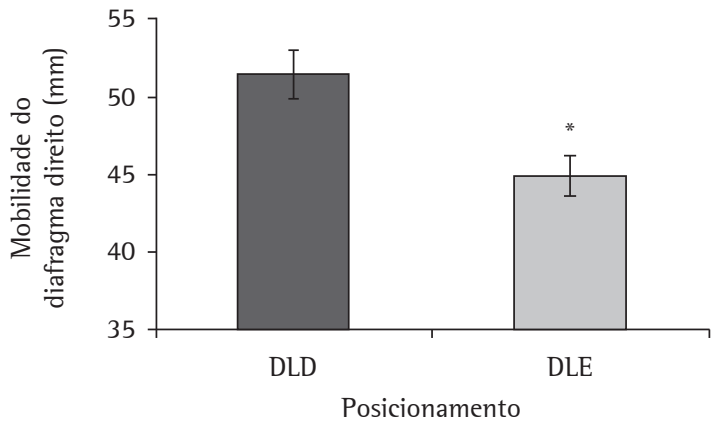

Figura 3 - Valores referentes à média da mobilidade do diafragma direito de sete sujeitos saudáveis nas posições decúbito lateral direito (DLD) e decúbito lateral esquerdo (DLE). *Diferença significativa $(p<0,05)$ entre os decúbitos.

Alguns mecanismos podem explicar o maior movimento do diafragma gerado nas zonas de maior pressão hidrostática durante a respiração espontânea. Na porção dependente, o peso das vísceras abdominais desloca o diafragma no sentido cefálico reduzindo o raio de curvatura do diafragma. ${ }^{(15)}$ Baseado na lei de Laplace, a pressão transdiafragmática nessa região tende a ser maior para a mesma tensão gerada pelo músculo diafragma.. ${ }^{(16)}$ Além disso, as fibras da hemicúpula do diafragma na porção dependente sofrem um alongamento pelo deslocamento cefálico, podendo, dessa forma, gerar maiores pressões e, conseqüentemente, maior movimento, devido à relação tensão-comprimento mais favorável. ${ }^{(17)}$ Outro fator a ser considerado é que na posição em decúbito lateral a mobilidade da porção dependente da caixa torácica encontra-se restrita, o que pode favorecer o deslocamento crânio-caudal do diafragma nessa região, já que o gradil costal 
assume uma função de ponto fixo para o movimento torácico durante o ciclo respiratório.(1)

Vários métodos por imagem têm sido utilizados para a avaliação da mobilidade diafragmática, dentre eles a fluoroscopia, a tomografia axial computadorizada, a ressonância nuclear magnética e a ultra-sonografia. A fluoroscopia tem sido considerada como o método mais confiável de avaliação quantitativa da amplitude do movimento crânio-caudal do diafragma durante a respiração espontânea. ${ }^{(18)} \mathrm{Na}$ avaliação pela fluoroscopia, a mobilidade do diafragma é mensurada utilizando o deslocamento do ponto mais alto das cúpulas diafragmáticas. Apesar de ser um método relativamente simples e permitir também a análise da ventilação pulmonar regional, essa técnica apresenta algumas desvantagens que devem ser consideradas: (i) exposição à radiação; (ii) amplitude da imagem devido à divergência dos raios- $x$, sendo necessária a realização de cálculos corretivos; e (iii) impossibilidade de obtenção de informação tridimensional do movimento diafragmático. ${ }^{(5)}$

Alguns autores têm recentemente utilizado a tomografia axial computadorizada e a ressonância nuclear magnética para realizar o estudo anatômico detalhado de diferentes porções do diafragma e da caixa torácica. ${ }^{(19,20)}$ Outros autores descreveram a mobilidade diafragmática em sujeitos normais utilizando imagens seqüenciais por ressonância magnética durante respiração basal. ${ }^{(21)}$ Esses autores discutem alguns aspectos positivos da utilização desse método na investigação do movimento diafragmático, como a possibilidade de digitalização das imagens, permitindo uma análise mais exata do movimento, e o fato de essa técnica não depender do investigador, minimizando variações de medidas inter e intra-observadores. Porém, o porte e custo dos equipamentos tornam esses métodos pouco práticos e menos disponíveis.

A ultra-sonografia tem sido mostrada como uma ferramenta promissora na avaliação da função diafragmática por ser um método simples, reprodutivel, portátil, sem risco de exposição à radiação ionizante e por quantificar diretamente o movimento do diafragma..$^{(22-24)}$ A visualização direta da cúpula diafragmática pode ser obtida posicionando-se o transdutor na linha média axilar entre os espaços intercostais, ${ }^{(7,9,10,25)}$ porém, nessa incidência a visualização do músculo torna-se difícil na inspiração profunda. ${ }^{(26)}$ Outros estudos utilizam a janela abdominal subcostal para a obtenção da imagem direta da cúpula diafragmática, ${ }^{(27)}$ contudo, nessa incidência o transdutor deve ser inclinado cranialmente para a visualização da porção posterior do diafragma ${ }^{(28)}$ e a excursão crânio-caudal do músculo se faz em direção oblíqua à incidência do feixe sonoro comprometendo assim a precisão da medida da mobilidade.

0 método ultra-sonográfico utilizado no presente estudo permite avaliar a mobilidade do hemidiafragma direito por meio de incidência do feixe sonoro perpendicular ao eixo crânio-caudal, utilizando janela abdominal subcostal durante todo o ciclo respiratório, mesmo na inspiração profunda. Apesar de esse método avaliar o deslocamento do ramo esquerdo da veia porta como medida indireta do deslocamento do diafragma direito, essa avaliação se faz sem as dificuldades metodológicas apresentadas anteriormente pelos outros métodos de avaliação ultra-sonográfica.

Uma limitação relatada por alguns autores que utilizam janela abdominal subcostal para avaliar a mobilidade diafragmática é a possivel interferência do transdutor sobre os movimentos abdominais durante a respiração. ${ }^{(20,24)}$ Esses autores discutem que a aplicação do transdutor sobre a parede abdominal pode modificar o padrão respiratório do sujeito, podendo comprometer a medida quando a avaliação é realizada durante a respiração basal. Acreditamos que essa influência pode ter sido menor no presente estudo, já que os sujeitos foram encorajados a realizar de maneira voluntária uma inspiração e expiração máxima, para mensurar o deslocamento das estruturas identificadas pelo avaliador.

0 cálculo do tamanho amostral no presente estudo foi estabelecido tendo como pressuposto os dados obtidos por alguns autores,${ }^{(2)}$ que constataram uma variação de $45 \%$ na mobilidade do diafragma com os diferentes posicionamentos e desvio padrão de $25 \%$ entre sujeitos. ${ }^{(2)}$ Porém, os resultados obtidos neste estudo mostraram uma variação da mobilidade diafragmática com os diferentes posicionamentos de 10,47\% e desvio padrão de 6,56\% entre sujeitos. Uma possível explicação para a discordância entre as variações pode ser devido ao fato de que, no estudo desses autores, ${ }^{(2)}$ a mobilidade diafragmática foi avaliada durante a ventilação com pressão positiva. Apesar das diferenças constatadas entre a variação obtida e a esperada, o tamanho amostral de sete sujeitos foi suficiente para detectar uma 
diferença significativa da mobilidade diafragmática nos diferentes posicionamentos.

Como citado anteriormente, alguns estudos têm avaliado a influência da mudança de postura corporal na mobilidade do diafragma em indivíduos em respiração espontânea ou em ventilação mecânica controlada. ${ }^{(2,3,29)}$ Devido às dificuldades de avaliação pela fluoroscopia, o número de pacientes avaliados por esses autores é sempre um fator limitante (três a quatro pacientes). Uma vantagem importante desse novo método é que a mobilidade do diafragma pode ser avaliada num curto espaço de tempo, sem apresentar riscos ao paciente e podendo ser realizada até mesmo à beira do leito. ${ }^{(24)}$ Esse método pode ter algumas aplicabilidades clínicas, tais como monitorar o impacto de doenças respiratórias e neuromusculares na mecânica diafragmática, quantificar benefícios de programas de reabilitação pulmonar direcionados ao treinamento diafragmático, ${ }^{(30)} \mathrm{e}$ avaliar a mobilidade do diafragma em pacientes com paralisia diafragmática.

Os nossos resultados sugerem que durante a ventilação espontânea a mobilidade da porção dependente do diafragma é maior do que a não dependente e que a técnica empregada foi sensível para detectar variações da mobilidade do diafragma com as mudanças de posicionamento. Esses resultados apresentam evidências que subsidiam o uso da ultra-sonografia na prática clínica para avaliar o deslocamento diafragmático.

\section{Referências}

1. Dean E, Ross J. Oxygen transport - The basis for contemporary cardiopulmonary physical therapy and its optimization with body position and mobilization. Physical Therapy Practice. 1992;1(4):34-44.

2. Froese AB, Bryan AC. Effects of anesthesia and paralysis on diaphragmatic mechanics in man. Anesthesiology. 1974;41(3):242-55.

3. Krayer S, Rehder K, Vettermann J, Didier EP, Ritman EL. Position and motion of the human diaphragm during anesthesia-paralysis. Anesthesiology. 1989;70(6):891-8

4. George RB, Weil H. Fluorodensimetry. A method for analyzing regional ventilation and diaphragm function. JAMA. 1971;217(2):171-6

5. Gierada DS, Slone RM, Fleishman MJ. Imaging evaluation of the diaphragm. Chest Surg Clin N Am. 1998;8(2):237-80

6. Harris RS, Giovannetti M, Kim BK. Normal ventilatory movement of the right hemidiaphragm studied by ultrasonography and pneumotachography. Radiology. 1983;146(1):141-4

7. Houston JG, Morris AD, Howie CA, Reid JL, McMillan N. Technical report: quantitative assessment of diaphragmatic movement - a reproducible method using ultrasound. Clin Radiol. 1992;46(6):405-7

8. Ayoub J, Cohendy R, Prioux J, Ahmaidi S, Bourgeois JM, Dauzat $\mathrm{M}$, et al. Diaphragm movement before and after cholecystectomy: a sonographic study. Anesth Analg. 2001;92(3):755-61

9. Houston JG, Fleet M, Cowan MD, McMillan NC. Comparison of ultrasound with fluoroscopy in the assessment of suspected hemidiaphragmatic movement abnormality. Clin Radiol. 1995;50(2):95-8

10. Houston JG, Angus RM, Cowan MD, McMillan NC, Thomson NC. Ultrasound assessment of normal hemidiaphragmatic movement: relation to inspiratory volume. Thorax. 1994;49(5):500-3

11. Toledo NS, Kodaira SK, Massarollo PC, Pereira Ol, Mies S. Right hemidiaphragmatic mobility: assessment with US measurement of craniocaudal displacement of left branches of portal vein. Radiology. 2003;228(2):389-94

12. Standardization of spirometry, 1994 Update. American Thoracic Society. Am J Respir Crit Care Med. 1995;152(3):1107-36

13. Sociedade Brasileira de Pneumologia e Tisiologia Consenso Brasileiro sobre Espirometria. J Pneumol. 1996;22(3):105-64

14. Knudson RJ, Lebowitz MD, Holberg CJ, Burrows B. Changes in the normal maximal expiratory flow-volume curve with growth and aging. Am Rev Resp Dis. 1983;127(6):725-34

15. Ibanez J, Raurich JM. Normal values of functional residual capacity in the sitting and supine positions. Intensive Care Med. 1982;8(4):173-7

16. Whitelaw WA, Hajdo LE, Wallace JA. Relationships among pressure, tension and shape of the diaphragm. J Appl Physiol. 1983;55(6):1899-905

17. Farkas GA, Roussos C. Diaphragm in emphysematous hamsters: sarcomere adaptability. J Appl Physiol. 1983;54(6):1635-40

18. Verschakelen JA, Deschepper K, Jiang TX, Demedts M. Diaphragmatic displacement measured by fluoroscopy and derived by respitrace. J Appl Physiol. 1989;67(2):694-8

19. Iwasawa T, Kagei S, Gotoh T, Yoshiike Y, Matsushita K, Kurihara $\mathrm{H}$, et al. Magnetic resonance analysis of abnormal diaphragmatic motion in patients with emphysema. Eur Respir J. 2002;19(2):225-31

20. Takazakura R, Takahashi M, Nitta N, Murata K. Diaphragmatic motion in the sitting and supine positions: healthy subject study using a vertically open magnetic resonance system. J Magn Reson Imaging. 2004;19(5):605-9

21. Gierada DS, Curtin JJ, Erickson SJ, Prost RW, Strandt JA, Goodman LR. Diaphragmatic motion: fast gradientrecalled-echo MR imaging in healthy subjects. Radiology. 1995;194(3):879-84

22. Gottesman E, McCool FD. Ultrasound evaluation of the paralyzed diaphragm. Am J Respir Crit Care Med. 1997;155(5):1570-4

23. Gerscovich EO, Cronan M, McGahan JP, Jain K, Jones CD, McDonald C. Ultrasonographic evaluation of diaphragmatic motion. J Ultrasound Med. 2001;20(6):597-604

24. Jiang JR, Tsai TH, Jerng JS, Yu CJ, Wu HD, Yang PC. Ultrasonographic evaluation of liver/spleen movements and extubation outcome. Chest. 2004;126(1):179-85

25. Wait JL, Nahormek PA, Yost WT, Rochester DP. Diaphragmatic thickness-lung volume relationship in vivo. J Appl Physiol. 1989;67(4):1560-8 
26. Gibson GJ. Diaphragmatic paresis: pathophysiology, clinical features and investigation. Thorax. 1989;44(11):960-70

27. Diament MJ, Boechat Ml, Kangarloo $\mathrm{H}$. Real-time sector ultrasound in the evaluation of suspected abnormalities of diaphragmatic motion. J Clin Ultrasound. 1985;13(8):539-43

28. Haber K, Asher M, Freimanis AK. Echographic evaluation of diaphragmatic motion in intra-abdominal diseases. Radiology. 1975;114(1):141-4
29. Akiyama N, lshikawa S, Takeuchi T. Ultrasonographic evaluation of the influence of different postures on diaphragmatic motion in mechanically ventilated patients. Eur J Ultrasound. 2000;11(3):205-11

30. Paulin E, Brunetto AF, Carvalho CRF. Efeitos de programa de exercícios físicos direcionado ao aumento da mobilidade torácica em pacientes portadores de doença pulmonar obstrutiva crônica. J Pneumol. 2003;29(5):287-94. 